\title{
Computerized ionospheric tomography with the IRI model
}

\author{
Orhan Arikan $^{\mathrm{a}}$, Feza Arikan ${ }^{\mathrm{b}, *}$, Cemil B. Erol $^{\mathrm{c}}$ \\ ${ }^{a}$ Bilkent University, Department of Electrical and Electronics Engineering, Bilkent Ankara 06800, Turkey \\ ${ }^{\mathrm{b}}$ Hacettepe University, Department of Electrical and Electronics Engineering, Beytepe Ankara 06800, Turkey \\ c TUBITAK - UEKAE, Kavaklidere, Ankara 06100, Turkey
}

Received 14 December 2005; received in revised form 6 February 2007; accepted 7 February 2007

\begin{abstract}
Computerized ionospheric tomography (CIT) is a method to estimate ionospheric electron density distribution by using the global positioning system (GPS) signals recorded by the GPS receivers. Ionospheric electron density is a function of latitude, longitude, height and time. A general approach in CIT is to represent the ionosphere as a linear combination of basis functions. In this study, the model of the ionosphere is obtained from the IRI in latitude and height only. The goal is to determine the best representing basis function from the set of Squeezed Legendre polynomials, truncated Legendre polynomials, Haar Wavelets and singular value decomposition (SVD). The reconstruction algorithms used in this study can be listed as total least squares (TLS), regularized least squares, algebraic reconstruction technique (ART) and a hybrid algorithm where the reconstruction from the TLS algorithm is used as the initial estimate for the ART. The error performance of the reconstruction algorithms are compared with respect to the electron density generated by the IRI-2001 model. In the investigated scenario, the measurements are obtained from the IRI-2001 as the line integral of the electron density profiles, imitating the total electron content estimated from GPS measurements. It has been observed that the minimum error between the reconstructed and model ionospheres depends on both the reconstruction algorithm and the basis functions where the best results have been obtained for the basis functions from the model itself through SVD.
\end{abstract}

(C) 2007 COSPAR. Published by Elsevier Ltd. All rights reserved.

Keywords: Ionosphere; Tomography; International Reference Ionosphere (IRI)

\section{Introduction}

Computerized ionospheric tomography (CIT) is becoming an attractive alternative in obtaining ionospheric electron density images. The global positioning system (GPS) makes it feasible to obtain measurements from various stations on the Earth with no additional cost to the users (Haij et al., 1994). The signals transmitted from the satellites in two L-band frequencies are collected by the Earth based receivers and recorded as pseudo-range and phase. Total electron content (TEC) is defined as the number of electrons included in a cylinder with $1 \mathrm{~m}^{2}$ cross-section. As is widely discussed in the literature, TEC, which corresponds

\footnotetext{
* Corresponding author.

E-mail addresses: oarikan@ee.bilkent.edu.tr (O. Arikan), arikan@ hacettepe.edu.tr (F. Arikan), cemil.erol@iltaren.tubitak.gov.tr (C.B. Erol).
}

to the line integral of the electron density in the path joining the satellite and the receiver, can be obtained from GPS receiver pseudo-range and phase measurements (Hocke and Pavelyev, 2001). With appropriate inversion and reconstruction methods, the electron density images can be obtained using the TEC measurements from the GPS receivers. This seemingly easy outline of obtaining electron density images actually requires complicated signal processing tools due to inherent errors in ionospheric electron density models, GPS measurements and TEC computation (Sutton and Na, 1998; Yeh and Raymund, 1991).

Ionospheric electron density is a function of latitude, longitude, height and time. A general approach in CIT is to represent the ionosphere as a linear combination of basis functions. In the literature, the ionosphere is usually represented by a linear combination of basis functions in two dimensions, namely latitude and height (Hajj et al., 
1994). In this study, following the examples in the literature, the model electron density distribution of the ionosphere is obtained from the International Reference Ionosphere (IRI) in latitude and height only. The best basis function set in height is determined to be eigenvectors from the singular value decomposition (SVD) of the IRI model in height (Sutton and Na, 1998). For the possible basis functions in latitude, Squeezed Legendre polynomials, Cut Legendre polynomials, Haar Wavelets and SVD of the IRI model in latitude are considered. The optimum number of basis functions in height and latitude are chosen under the minimum error criterion of reconstructed images. The 2-D CIT is performed by using a set of certain reconstruction algorithms, namely total least squares (TLS), regularized least squares (RLS), algebraic reconstruction technique (ART) and a hybrid algorithm (HART) where the reconstruction from the TLS algorithm is used as the initial estimate for the iterations of ART (Yavuz et al., 2005a,b). The performance of the reconstruction algorithms and basis functions are tested in a scenario in the $\left[-28^{\circ}\right.$ to $\left.28^{\circ}\right]$ latitude interval. Ionosphere is divided into a grid formed by rectangular pixels. It is assumed that, electron density has uniform distribution in each pixel. As an initial step in the overall investigation of the performance of various CIT techniques, the TEC estimates from the IRI are used. Thus, the measurement vector is obtained from the line integral of the electron density of the IRI model over the constructed grid. For the chosen ideal scenario, the reconstruction error is obtained as low as 0.2 for SVD bases both in height and latitude used with TLS and RLS algorithms. The computational complexity of TLS is lower than RLS. ART is independent of basis functions and very sensitive to the initial state. The highest error is observed when the squeezed Legendre polynomials are used in latitude.

In Section 2, the general set up of the electron density reconstruction model is provided. The basis functions and reconstruction algorithms are briefly discussed in Section 3 and the results of the study are presented in Section 4.

\section{CIT model}

CIT is method of reconstruction of ionospheric electron density from GPS measurements. Since ionosphere varies with respect to position (latitude and longitude), height from earth's surface and time, the formulation of CIT in four dimensions is highly complicated. In this section, the ionospheric electron density is represented as a linear combination of basis functions in latitude and height. Our goal is to test the accuracy and computational complexity of the various possible orthonormal basis function sets and reconstruction algorithms in a reduced dimension system. In this study, $g(r, \theta)$ represents the ionospheric electron density profile in height, $r$, and in angle measured from global zenith, $\theta$, corresponding to $90^{\circ}$-latitude, where latitude takes positive values in northern hemisphere and negative values in southern hemisphere. Let us define a grid structure which expands the region of interest in ionosphere. A pixel in the grid is defined by the pair $\left(n_{r}, n_{\theta}\right)$, where $n_{r}=1, \ldots, N_{r}$ and $n_{\theta}=1, \ldots, N_{\theta}$. Any sample of $g(r, \theta)$ at $\left(n_{r}, n_{\theta}\right)$ can be given as $g_{s}\left(n_{r}, n_{\theta}\right)$ where the subscript $s$ denotes the sampled electron density profile at the grid pixel $\left(n_{r}, n_{\theta}\right)$. Instead of using two dimensions as grid pixel notation, a lexicographical index $l$ can be defined as $l=n_{r}+\left(n_{\theta}-1\right) N_{r}$ and the sampled electron density can be given as $g_{s}\left(n_{r}, n_{\theta}\right)=g_{s}(l)$. This notation helps us to reduce computational dimension from two to one. The model electron density vector using the index $l$ can be defined as

$\mathbf{g}=\left[g_{s}(1) \ldots g_{s}(l) \ldots g_{s}\left(N_{r} N_{\theta}\right)\right]_{1 \times N_{r} N_{\theta}}^{\mathrm{T}}$

where the superscript $\mathrm{T}$ denotes the transpose.

In serial expansion method, the electron density profile $g(r, \theta)$ is approximated by a finite number of 2-D basis functions with unknown coefficients as follows:

$$
\begin{aligned}
g(r, \theta) \approx \hat{g}(r, \theta ; M, N)= & \sum_{m=1}^{M} \sum_{n=1}^{N} \alpha_{m, n} u_{m}(r) v_{n}(\theta) \\
& =\sum_{k=1}^{K} \alpha_{k} \phi_{k}(r, \theta)
\end{aligned}
$$

where $M$ and $N$ represent the number of basis functions $u_{m}(r)$ in height and $v_{n}(\theta)$ in latitude, respectively. With shorthand notation, $\phi_{k}(r, \theta)=u_{m}(r) v_{n}(\theta)$ where $k=m+(n-1) M$ and $K=M N$. The tomography problems are usually formulated in a linear system and the coefficients $\alpha_{m, n}$ or $\alpha_{k}$ are the unknowns to be determined in the reconstruction problem.

For a pixel $\left(n_{r}, n_{\theta}\right)$, denoted by the index $l$ in the grid, the samples of the basis functions $\phi_{k}$ can be expressed as $\phi_{k s}(l)=u_{m s}\left(n_{r}\right) v_{n s}\left(n_{\theta}\right)$, where $u_{m s}$ and $v_{n s}$ are the sampled height and latitude basis functions, respectively. From the sampled basis functions, the vector $\underline{\phi}_{k}$ is defined as

$\underline{\phi_{k}}=\left[\phi_{k s}(1) \ldots \phi_{k s}(l) \ldots \phi_{k s}\left(N_{r} N_{\theta}\right)\right]_{1 \times N_{r} N_{\theta}}^{\mathrm{T}}$

and the sampled ionospheric electron density function can be rewritten as

$\mathbf{g}(l) \approx \sum_{k=1}^{K} \alpha_{k} \underline{\phi}_{k}$

where $\mathbf{g}(l)$ corresponds to the $l$ th member of $\mathbf{g}$ in Eq. (1) and thus is equal to $g_{s}\left(n_{r}, n_{\theta}\right)$.

In the tomographic reconstruction scenario in this study, the measurements are obtained as the line integrals of the electron density profiles or the summations of the samples of ionospheric electron density function. Let the number of measurements be denoted as $n_{m}=1, \ldots, N_{m}$, and the measurement on the $n_{m}^{\text {th }}$ projection to be $d_{n_{m}}$ where

$d_{n_{m}}=\sum_{l=1}^{N_{r} N_{\theta}} \beta_{n_{m}, l} g_{s}(l)$ 
and $\beta_{n_{m}, l}$ is equal to 1 if the $l$ th pixel is on the $n_{m}^{\text {th }}$ projection; and 0 otherwise. Then, we can define a matrix $\mathbf{B}\left(n_{m}, l\right)=\beta_{n_{m}, l}$ and the measurement vector $\mathbf{d}=\left[d_{1} \ldots d_{n_{m}} \ldots d_{N_{m}}\right]^{\mathrm{T}}$ using Eq. (6) as

$\mathbf{d}_{N_{m} \times 1}=\mathbf{B}_{N_{m} \times N_{r} N_{\theta}} \mathbf{g}_{N_{r} N_{\theta} \times 1}$.

If the serial expansion in Eq. (5) is used in the above measurement equation, the measurement vector can be rewritten as

$\mathbf{d} \approx \sum_{k=1}^{K} \underbrace{\mathbf{B} \phi_{k}}_{\mathbf{p}_{k}} \alpha_{k}$.

Eq. (8) can be expressed in a more closed form matrix notation as

$\mathbf{d}=\mathbf{P} \underline{\alpha}$,

where

$\mathbf{P}=\left[\mathbf{p}_{1} \cdots \mathbf{p}_{K}\right]_{N_{m} \times K}$

and

$\underline{\alpha}=\left[\alpha_{1} \cdots \alpha_{K}\right]^{\mathrm{T}}$.

Eq. (9) translates the 2-D reconstruction problem of $g\left(r_{n_{r}}, \theta_{n_{\theta}}\right)$ to a 1-D reconstruction problem in terms of basis coefficients $\underline{\alpha}$. Eq. (9) is in the form of a typical linear systems problem. $\mathbf{P}$ denotes the model ionosphere in the form of basis functions, $\mathbf{d}$ represents the measurement vector and $\underline{\alpha}$ are the unknown coefficients to be determined by the solution of the linear system of equations. There are various alternatives for the basis functions to be used in the serial expansion and the number of total basis functions is a parameter to be determined for a given scenario. Usually, the model matrix $\mathbf{P}$ is not full rank or the model includes certain errors. The measurements can be noisy and sparse to properly represent the electron density. Therefore, the solution of Eq. (9) is a challenge, where computational complexity and accuracy of the solutions are important performance criteria to be considered. In the next section, we will discuss possible alternatives to solve this problem and choices of basis functions in height and latitude.

\section{Basis functions and reconstruction algorithms}

The performance of CIT depends on both the number and choice of basis functions in height and latitude and the reconstruction algorithms to be implemented to solve the linear system problem (Fremouw and Secan, 1992). Using the notation of Section 2, the sampled model electron density matrix defined over the grid $N_{r} \times N_{\theta}$ can be given as

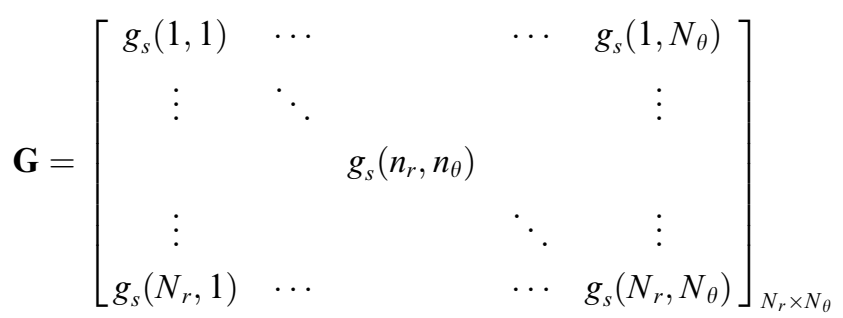

In height and in latitude, the natural basis is obtained by the singular value decomposition of the electron density matrix $\mathbf{G}$ as

$\mathbf{G}=\mathbf{U} \boldsymbol{\Sigma} \mathbf{V}^{H}$

where the superscript $H$ denotes the Hermitian. $\boldsymbol{\Sigma}$ is a diagonal matrix containing the singular values $\sigma$ in decreasing order. The matrix $\mathbf{U}$ contains left singular vectors and $\mathbf{V}$ has the right singular vectors. If the singular values in $\mathbf{\Sigma}$ are plotted, it is observed that most of the energy is captured in first few singular values. Thus, the singular value decomposition of $\mathbf{G}$ can be approximated as

$\mathbf{G} \approx \sum_{n_{s}=1}^{N_{s}} \sigma_{n_{s}} \mathbf{u}_{n_{s}} \mathbf{v}_{n_{s}}^{H}$.

where $N_{s}$ is the number of significant singular values $\sigma_{n_{s}}$. Here, the vectors $\mathbf{u}_{n_{s}}$ and $\mathbf{v}_{n_{s}}$ denote the first $N_{s}$ left and right singular vectors of $\mathbf{G}$, respectively. Then, the basis functions in Eq. (2) are formed using $\mathbf{u}_{n_{\mathrm{s}}}$ in height and $\mathbf{v}_{n_{s}}$ in latitude.

Another commonly used basis in CIT is obtained from the Legendre functions of the first kind, which are also called as Legendre polynomials. Legendre polynomials, $P_{n}(x)$, where $-1<x<1$, are solutions to the ordinary Legendre differential equation. Here, $n$ is an integer denoting the order and $x$ is the argument. When the second order differential equation is represented in spherical coordinates, the total differential equation in $\theta$ turns out to be Legendre differential equation whose solution can be expressed as Legendre polynomials with argument $x=\cos \theta$. The range of $\theta$ in spherical coordinates is $0<\theta<\pi$. Thus, $P_{n}(\cos \theta)$ constitutes a natural orthonormal basis set in latitude if the region of interest extends from $0<\theta<\pi$. For any other region extending from $\theta_{i}$ to $\theta_{N_{\theta}}$, the polynomials have to be modified to form a new orthonormal basis in the region of interest. One possible way is to scale the polynomials so that the squeezed polynomials form an orthogonal set in the region of interest. Further, the squeezed polynomials have to be orthonormalized by using the Gram-Schmidt algorithm (Yavuz et al., 2005b). Another way is to truncate the Legendre functions in the region extending from $\theta_{i}$ to $\theta_{N_{\theta}}$. Since the truncated polynomials will not be orthonormal, Gram-Schmidt algorithm will again have to be used to obtain orthonormalized Truncated Legendre bases in latitude (Yavuz et al., 2005b).

Wavelets with their multi-scale structure are plausible alternatives in the selection of latitude basis functions. 
Haar Wavelets are very easy to generate and implement in any region of interest in latitude (Yavuz et al., 2005a,b). Other possible wavelet bases to be used in CIT reconstructions include spherical Haar and Mexican Hat wavelets.

To obtain robust and computationally efficient estimates to the electron density distributions, it is important to use only few number of basis vectors.

The tomographic reconstruction algorithms vary in terms of their computational complexity and accuracy. One of the simplest algorithms is Algebraic Reconstruction Technique (ART) which does not require any explicitly defined basis functions (Austen et al., 1988; Roerdink, 1992). However, being an iterative technique, it is highly dependent on the starting estimate of the electron density distribution.

Regularized least squares (RLS) brings a regularization to the basic least squares solution as

$\hat{\alpha}_{\text {RLS }}=\left(\mathbf{P}^{\mathrm{H}} \mathbf{P}-\mu \mathbf{I}\right)^{-1} \mathbf{P}^{\mathbf{H}} \mathbf{d}$,

where $\mu$ is the regularization coefficient that should be chosen carefully according to the nature of the problem (Golub et al., 2000).

Total least squares (TLS) algorithm assumes that there are uncertainties in both the model ionosphere matrix and the measurement vector in Eq. (9), and estimates the unknown coefficients as

$\underline{\hat{\alpha}}_{\mathrm{TLS}}=\left(\mathbf{P}^{\mathrm{H}} \mathbf{P}-\sigma^{2} \mathbf{I}\right)^{-1} \mathbf{P}^{\mathrm{H}} \mathbf{d}$,

where $\sigma$ is the smallest non-zero singular value of the augmented matrix $[\mathbf{P} \mid \mathbf{d}]$ and $\mathbf{I}$ is the identity matrix. The computational complexity and accuracy of the above mentioned algorithms are compared in the next section as they are applied to an example scenario.

\section{Results}

The RLS, TLS and HART reconstruction algorithms are applied with SVD, Squeezed Legendre, Truncated Legendre and Haar Wavelet bases to an ionospheric electron density reconstruction problem. A CIT scenario is constructed to compare the computational complexity of the basis functions and reconstruction algorithms. The reference electron density is obtained from the IRI-2001 model for $\left[-28^{\circ}\right.$ to $\left.28^{\circ}\right]$ latitude interval. The input parameters of IRI-2001 for this scenario are provided in Table 1 and the contour plot of the electron density is given in

Table 1

Input parameter set of IRI-2001 for the chosen CIT scenario

\begin{tabular}{ll}
\hline Parameter & Value \\
\hline Year, Month, Day, Hour & $2003,08,05,15.5$ \\
Longitude & $34^{\circ} \mathrm{E}$ \\
Solar zenith angle & $66.5^{\circ}$ \\
Magnetic inclination (dip) & $-61.56^{\circ}$ \\
Modified dip (modip) & $-48.83^{\circ}$ \\
Solar Sun spot number (Rz12) & 60.6 \\
Ionospheric-effective Solar index (IG12) & 71 \\
\hline
\end{tabular}

Fig. 1. Ionosphere is divided into 95 pixels in the vertical direction and 29 pixels in the latitude direction. Height of each pixel is $10 \mathrm{~km}$, and the width of each pixel is $2^{\circ}$. The projections are collected at $0^{\circ}$ in $\left[-28^{\circ} 28^{\circ}\right]$ latitude interval. There are 57 projections equally distributed in latitude. Electron density in each pixel is assumed to have a uniform distribution. Ionosphere is assumed to be time invariant during the computation.

The reconstructed electron density on the grid can be defined as

$\hat{\mathbf{G}}_{s}(N, M)=\sum_{m=1}^{M} \sum_{n=1}^{N} \hat{\alpha}_{m, n} u_{m s}\left(n_{r}\right) v_{n s}\left(n_{\theta}\right)$

where $\hat{\alpha}_{m, n}=\hat{\alpha}(m+(n-1) M)$ represent the estimated unknown basis function coefficients.

Once an estimate, $\underline{\hat{\alpha}}$, for the basis coefficients is obtained, the normalized reconstruction error, $\epsilon(N, M)$, is defined as

$\epsilon(N, M)=\frac{\left\|\mathbf{G}-\hat{\mathbf{G}}_{s}(N, M)\right\|}{\|\mathbf{G}\|}$

where $\|\cdot\|$ denotes the $\mathscr{L}_{2}$ norm.

The optimum number of latitude and vertical basis functions are important parameters in performance of the reconstruction algorithms. The optimum number of vertical basis functions are determined by examining the SVD of the ionospheric electron density profile $\mathbf{G}$ in height direction. The optimum number in height is found to be 3 for the examined scenario. The optimum number of basis functions in the latitude direction, $N_{o}$, is determined by examining the reconstruction error in Eq. (18), $\epsilon\left(N_{\mathrm{o}}, 3\right)$. The reconstruction error drops sharply as the number of basis functions increase and settles to a limiting value where further increasing the basis number does not change the error significantly. The optimum number of basis functions in the latitude, $N_{\mathrm{o}}$, is determined as the point where the error does not change significantly as the number of basis functions increase. For the chosen scenario, all the above mentioned reconstruction algorithms and basis function sets are tried for the lowest possible error and the optimum number of latitude basis functions in each case is recorded. In Table 2, a summary of best error performance for a selection of basis functions and reconstruction algorithms are provided when SVD basis is chosen for the height. As can be observed from the results, the minimum number of basis functions in latitude is obtained for SVD basis of 3 .

In Table 2, the overall reconstruction error for the optimum number of basis functions in both height and latitude, $\epsilon\left(N_{\mathrm{o}}, 3\right)$, is also provided for various reconstruction algorithms. The contour map of the electron density distribution obtained from using RLS as the reconstruction algorithm, SVD basis functions in height and squeezed Legendre bases in latitude is provided in Fig. 2. The squeezed Legendre bases actually gives the highest error for all reconstruction algorithms. Thus, as expected, using Legendre polynomials as they are within the region of interest results in very high error and high computational 


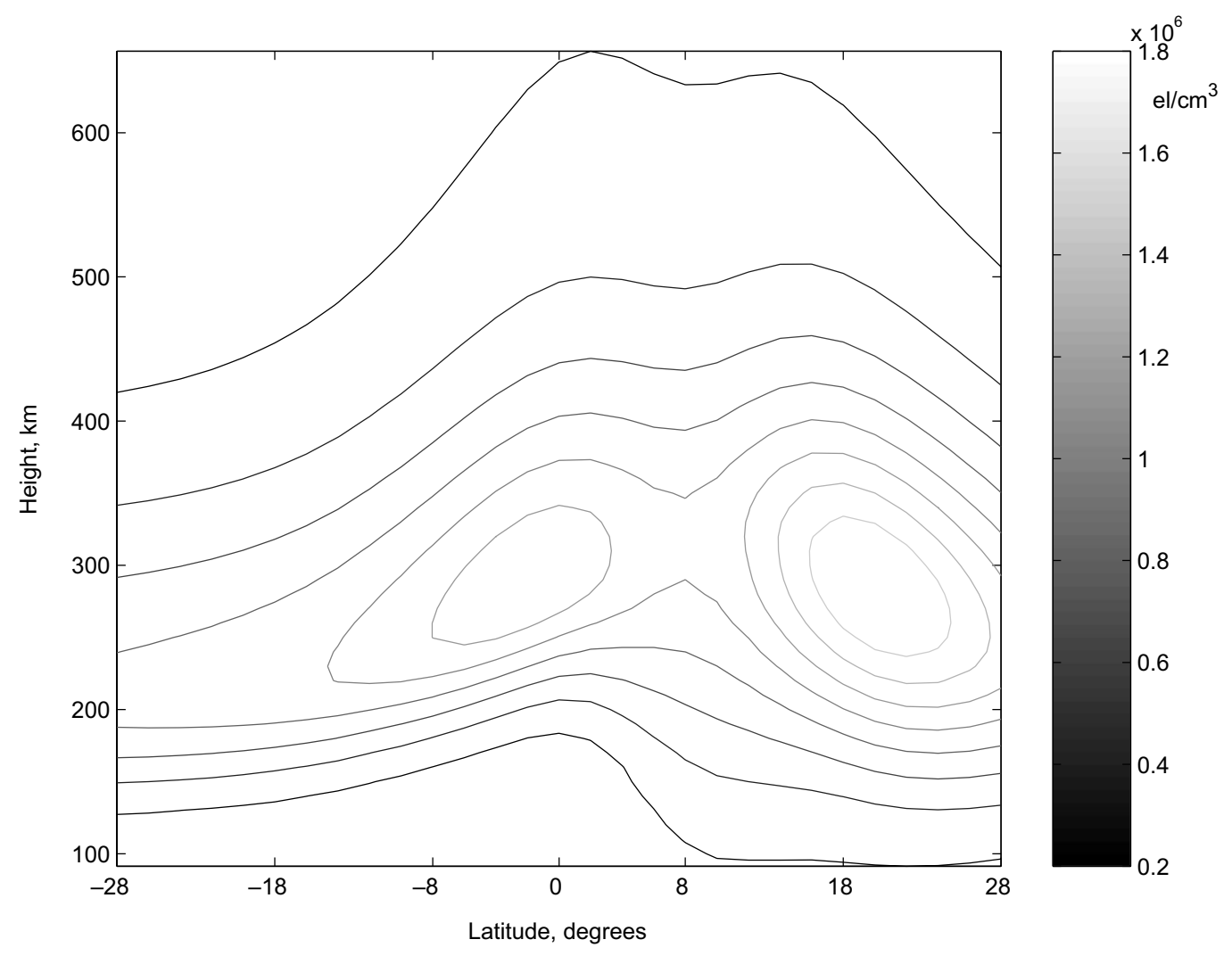

Fig. 1. The contour map of the electron density distribution obtained from IRI-2001 model for the region and dates given in Table 1 .

Table 2

The optimum reconstruction error performance for certain algorithms and basis functions for the chosen CIT scenario

\begin{tabular}{llll}
\hline Reconstruction algorithm & Basis function in latitude & $N_{\mathrm{o}}$ & $\epsilon\left(N_{\mathrm{o}}, 3\right)$ \\
\hline RLS & Squeezed Legendre & 34 & 0.5511 \\
ART & - & - & 0.3324 \\
RLS & Truncated Legendre & 32 & 0.2454 \\
TLS & Haar Wavelets & 26 & 0.2206 \\
HART & - & - & 0.2191 \\
TLS & SVD & 3 & 0.1937 \\
RLS & SVD & 3 & 0.1934 \\
\hline
\end{tabular}

complexity. As stated in the previous section, ART does not require basis functions and it is an iterative method. In Fig. 3, the contour map of the electron density distribution reconstruction using ART is given. Although it is lower in computational complexity, the error is highly dependent on the initial state. If the iteration is started with an uneducated guess such as a zero matrix, the reconstruction error increases to unacceptable levels. Truncated Legendre bases with TLS or RLS algorithms reduces the reconstruction error to almost half of the squeezed Legendre bases errors. Thus, if Legendre polynomials are truncated to the region of interest and orthonormalized, the reconstruction error reduces significantly. Using wavelet basis further reduces the error for both RLS and TLS. Haar Wavelets are very easy to generate and implement to the desired region of interest. The optimum numbers of latitude bases for Haar Wavelets for RLS and TLS are also lower than those for the Legendre bases. The contour map of the electron density distribution obtained from using TLS as the reconstruction algorithm, SVD basis functions in height and Haar Wavelet bases in latitude is given in Fig. 4. When the reconstructed electron density distribution in Fig. 4 is used as the initial state of the ART algorithm, a fast and low computationally complex reconstruction is obtained with reduced error as given in Table 2. The lowest number of optimum bases in height and latitude and the best error performance is obtained when SVD bases is used. Only for $K=9$ or $M=3$ and $N_{\mathrm{o}}=3$, the lowest error in reconstruction is obtained both for RLS and TLS algorithms. The contour map of the electron density distribution obtained from using regularized least squares as the reconstruction algorithm, SVD basis functions in both height and latitude is given in Fig. 5. Since SVD provides the natural basis for the region of interest, the reconstructions are very successful even though the projections are collected at only one position. As the number of projections and collection positions increase, it is expected that the reconstruction errors will further decrease.

\section{Conclusions and future work}

In this study, the error performance of RLS, TLS, ART and HART algorithms are investigated when they are used with SVD bases in height and Squeezed Legendre, 

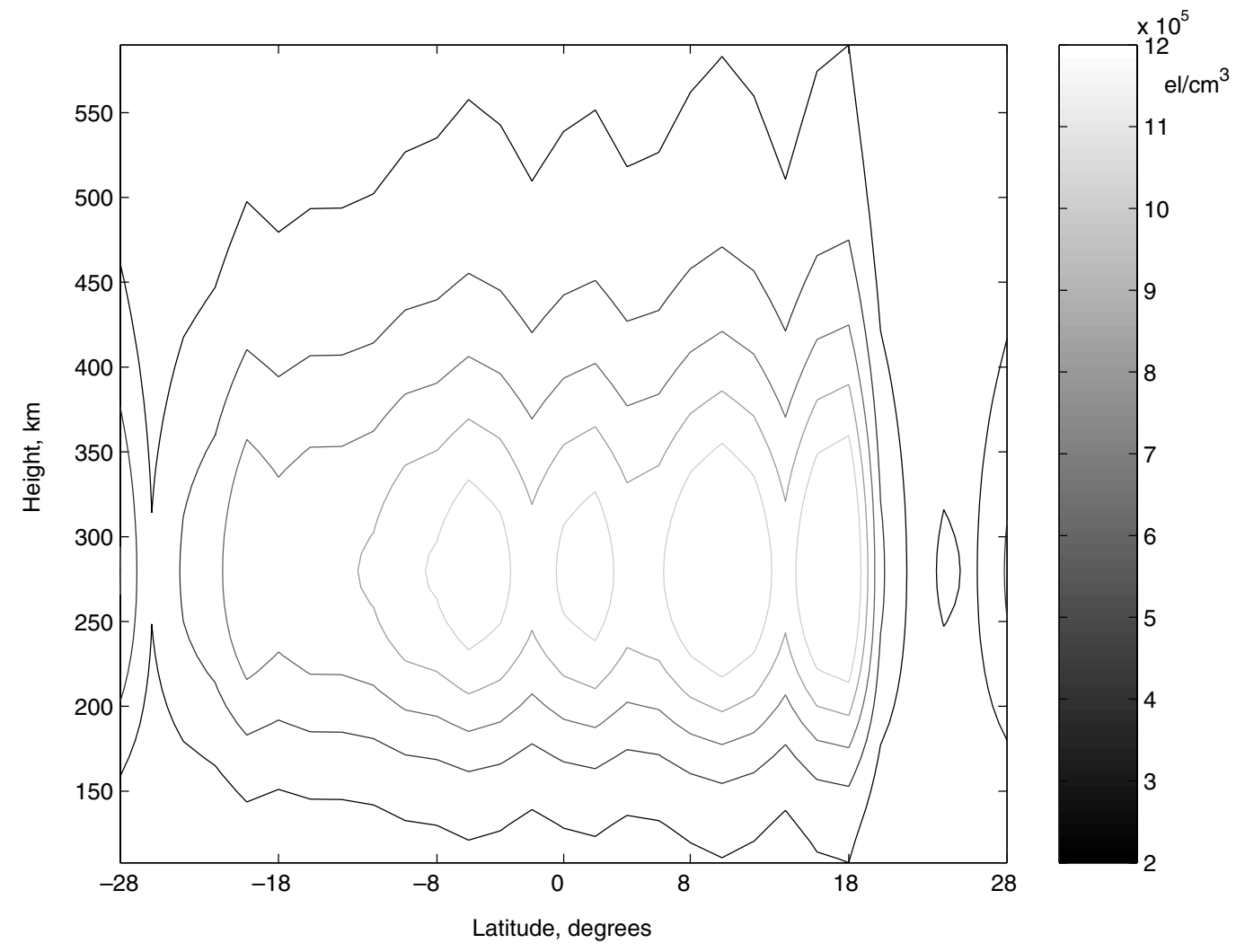

Fig. 2. The contour map of the electron density distribution obtained from using regularized least squares as the reconstruction algorithm, 3 SVD basis functions in height and 34 squeezed Legendre bases in latitude.

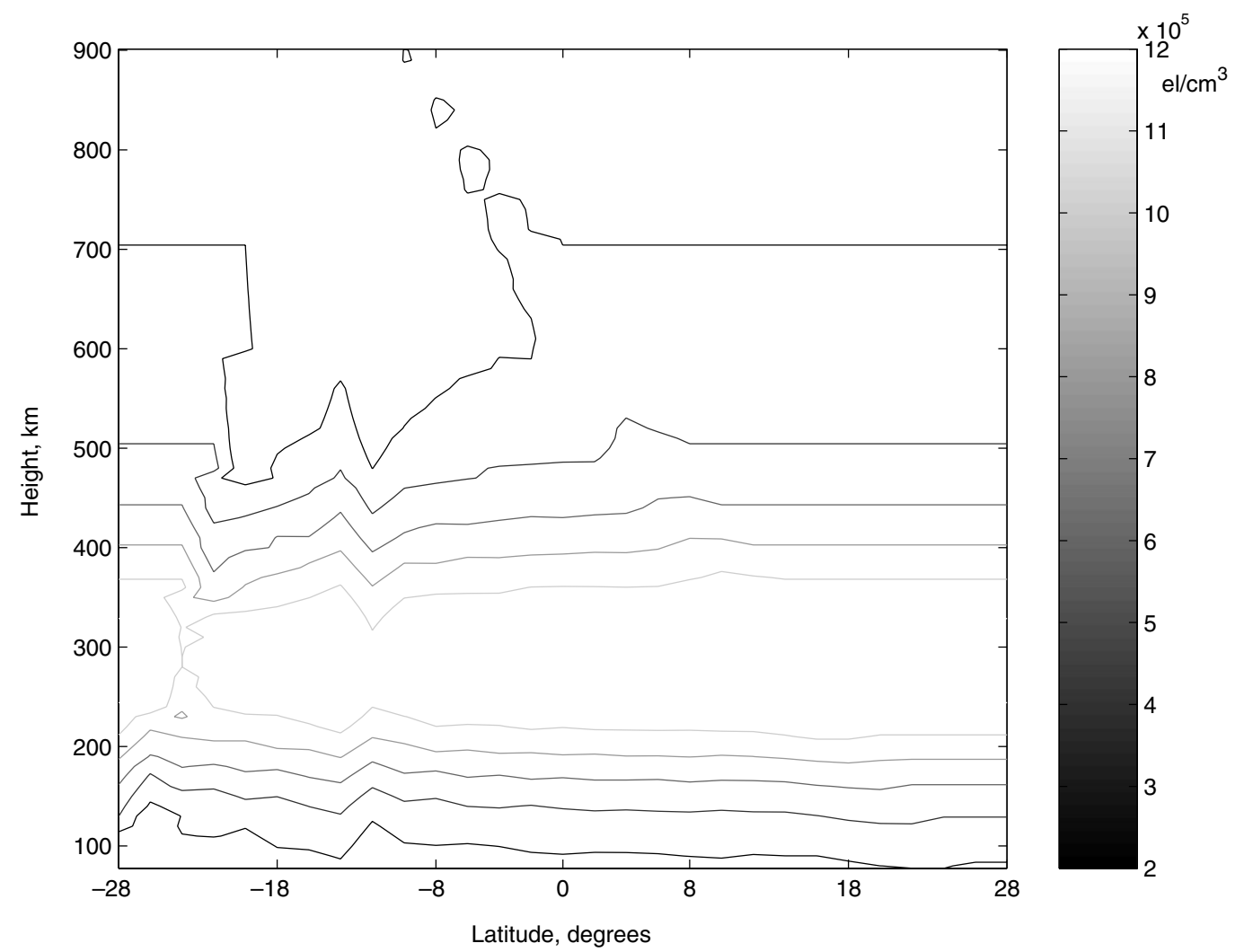

Fig. 3. The contour map of the electron density distribution obtained from using algebraic reconstruction technique which does not require any bases. 


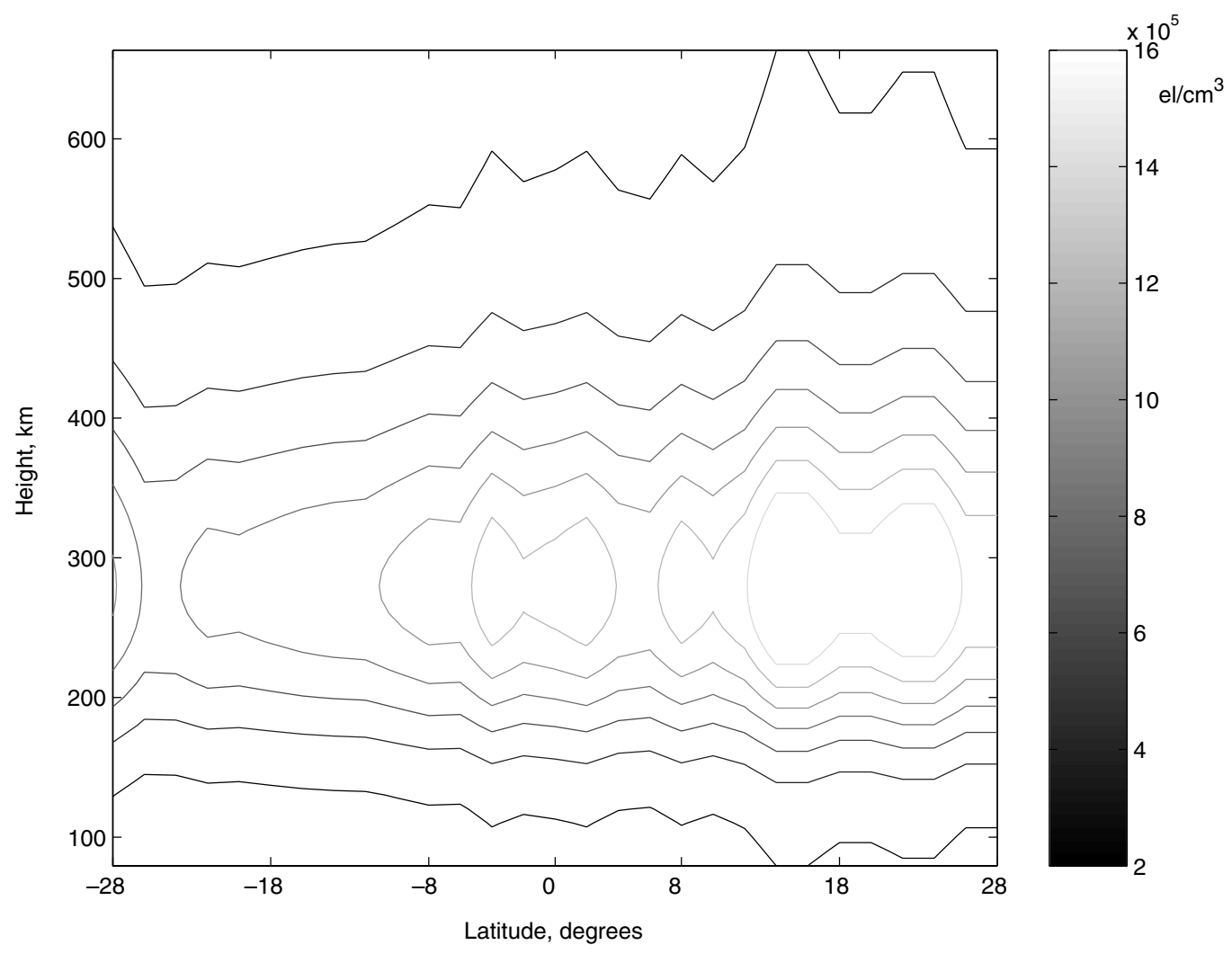

Fig. 4. The contour map of the electron density distribution obtained from using total least squares as the reconstruction algorithm, 3 SVD basis functions in height and 26 Haar Wavelet bases in latitude.

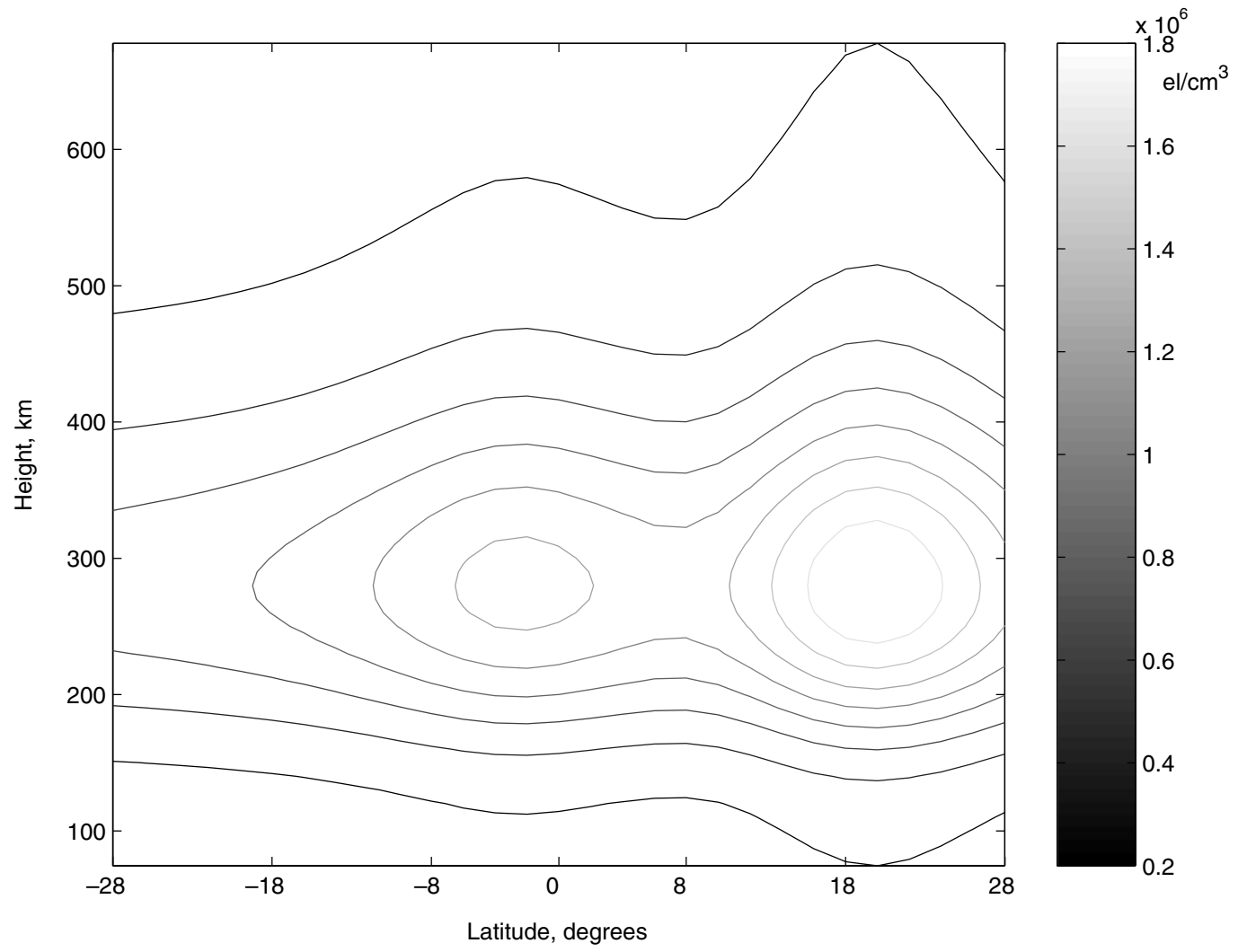

Fig. 5. The contour map of the electron density distribution obtained from using regularized least squares as the reconstruction algorithm, 3 SVD basis functions in height and 3 SVD basis functions in latitude. 
Truncated Legendre, Haar Wavelet and SVD bases in latitude for an example scenario. The IRI-2001 is used as the reference model in the serial expansion method and the measurements are obtained again from IRI-2001 by integrating the electron density in the pixels on the ray path. The reconstruction errors for the optimum number of latitude basis functions are provided for the chosen scenario. It is observed that SVD bases, which are the natural bases of the chosen distribution, provide the most successful reconstruction with the least computational complexity. As the number of bases increases, the reconstruction error decreases. The iterative algorithms such as ART are very sensitive to the initial state. When the initial state is chosen as the reconstruction of electron density distribution using, for example, TLS algorithm and Haar Wavelet basis functions, the reconstruction error in ART can be reduced significantly. It is expected that the reconstruction error to be further reduced when multiple satellites and receivers are used. In the future, this study will be extended to other latitude ranges and for various states of the ionosphere. The synthetic data will be replaced by the TEC values computed from the GPS recordings. The analytic expressions will be derived for multiple satellite and receiver cases.

\section{Acknowledgements}

This work is supported by TÜBITAK EEEAG Grant 105E171. The authors thank to Mr. E. Yavuz of Ziraat
Bankas1, Tandoğan, Ankara, Turkey for his help in producing figures.

\section{References}

Austen, J.R., Franke, S.J., Liu, C.H. Ionospheric imaging using computerized tomography. Radio Sci. 23 (3), 299-307, 1988.

Fremouw, E.J., Secan, J.A. Application of stochastic inverse theory to ionospheric tomography. Radio Sci. 27 (5), 721-732, 1992.

Golub, G.H., Hansen, P.C., O'Leary, D.P. Tikhonov regularization and total least squares. SIAM J. Matrix Anal. Appl. 21, 185-194, 2000.

Hajj, G.A., Ibanez-Meier, R., Kursinski, E.R. Imaging the ionosphere with the global positioning system. Int. J. Imag. Syst. Tech. 5, 174-184, 1994.

Hocke, K., Pavelyev, A.G. General aspect of GPS data use for atmospheric science. Adv. Space Res. 27 (6-7), 1313-1320, 2001.

Roerdink, J.B.T.M. Computerized tomography and its applications: a guided tour. Nieuw Archief voor Wiskunde 10 (3), 277-308, 1992

Sutton, E., Na, H. Time-varying reconstruction of the ionosphere. Int. J. Imag. Syst. Tech. 9, 484-490, 1998.

Yavuz, E., Arıkan, F., Arıkan, O.A. Hybrid reconstruction algorithm for computerized ionospheric tomography. In: Proceedings of RAST-2005, Recent Advances in Space Research, Harbiye Askeri Muze, Hava Harb Okulu, Istanbul, Turkey, 9-11 July, pp. 782787, 2005a.

Yavuz, E., Arıkan, F., Arıkan, O., Erol, C.B. Algorithms and basis functions in tomographic reconstruction of ionosphere electron density. In: Proceedings of EUSIPCO'2005, 13th European Signal Processing Conference, Antalya, Turkey, 4-8 September, 2005b.

Yeh, K.C., Raymund, T.D. Limitations of ionospheric imaging by ionospheric tomography. Radio Sci. 26 (6), 1361-1380, 1991. 